\title{
Low-Dimensional Models for Control of Leading-Edge Vortices: Equilibria and Linearized Models
}

\author{
Sunil Ahuja, Clarence W. Rowley†, Ioannis G. Kevrekidis; Mingjun Wei ${ }^{\ddagger}$ \\ Princeton University, Princeton, NJ 08544 \\ Tim Colonius, \\ Department of Mechanical Engineering, Caltech, Pasadena, CA 91125 \\ Gilead Tadmor" \\ Northeastern University, Boston, MA 02115
}

\begin{abstract}
When an airfoil is pitched up rapidly, a dynamic stall vortex forms at the leading edge and produces high transient lift before shedding and stall occur. The aim of this work is to develop low-dimensional models of the dynamics of these leading-edge vortices, which may be used to develop feedback laws to stabilize these vortices using closed-loop control, and maintain high lift. We first perform a numerical study of the two-dimensional incompressible flow past an airfoil at varying angles of attack, finding steady states using a timestepper-based Newton/GMRES scheme, and dominant eigenvectors using ARPACK. These steady states may be either stable or unstable; we develop models linearized about the stable steady states using a method called Balanced Proper Orthogonal Decomposition, an approximation of balanced truncation that is tractable for large systems. The balanced POD models dramatically outperform models using the standard POD/Galerkin procedure, and are used to develop observers that reconstruct the flow state from a single surface pressure measurement.
\end{abstract}

\section{Introduction}

Leading edge vortices have been studied extensively in the past few decades, largely in the context of helicopter rotors, ${ }^{1}$ but the idea of active control of these vortices, for instance using air injection, is relatively new. Several recent studies have explored modeling these flows for control purposes, using phenomenological models based on empirical data ${ }^{2}$ or vortex models. ${ }^{3,4}$ The goal of this work is to gain a better understanding of the dynamics of these flows first through a study of the steady states for different angles of attack, and then through low-dimensional models linearized about these equilibria.

Experimental studies on pitching airfoils demonstrate that when the angle of attack is varied rapidly, transient periods of high lift are observed, as sketched in Fig. 1 (left). As the angle of attack is varied periodically, the lift is greater when the angle of attack is increasing, and lower when the angle of attack is decreasing. ${ }^{5}$ This hysteresis phenomenon is relatively well understood from an intuitive point of view: a leading-edge vortex forms, causing high lift, but then sheds, and the lift decreases once again. However, existing dynamical models treat the hysteresis loop as a simple first- or second-order lag in reaching the steady state value. For instance, for low Reynolds numbers and/or low aspect ratio wings, such as insect wings, the leading-edge vortices have been shown to be stable. ${ }^{6}$ This implies that as the Reynolds number or

*Ph.D. Candidate, Mechanical and Aerospace Engineering, Student Member

${ }^{\dagger}$ Assistant Professor, Mechanical and Aerospace Engineering, Member AIAA

$\ddagger$ Professor, Chemical Engineering

$\S$ Postdoctoral scholar, Mechanical and Aerospace Engineering, Member AIAA

ๆ Professor, Mechanical Engineering, Member AIAA

" Professor, Electrical and Computer Engineering, Member AIAA

Copyright (C) 2007 by the authors. Published by the American Institute of Aeronautics and Astronautics, Inc. with permission. 

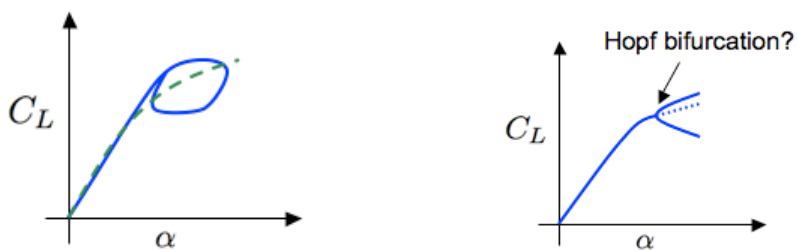

Figure 1. Experiments with airfoils at periodically varying angles of attack $(\alpha)$ show hysteresis loops as depicted at left, where the dashed curve indicates the steady-state lift values. The figure on the right shows a possible interpretation of this phenomenon as a Hopf bifurcation; here stable equilibrium points or periodic orbits are shown as solid lines, and unstable equilibria as dashed ones.

the aspect ratio increases, a loss of stability occurs, which manifests itself as a shedding of the leading-edge vortex and a loss in lift. However, from a dynamical systems perspective, one expects that the (now unstable) steady state is still present, and one might use feedback to stabilize this high-lift equilibrium. For instance, in Fig. 1 (right) a cartoon of a possible bifurcation diagram is shown, in which the stable equilibria or periodic orbits are shown by solid lines, and an unstable branch is indicated by a dashed line. The ultimate goal is to identify any unstable, high-lift equilibria, to develop models for the dynamics near these equilibria, incorporating the effects of actuators, and finally to design feedback controllers to stabilize these equilibria.

The specific goals of this paper are to identify steady states (steady solutions of the Navier-Stokes equations for this geometry), and develop linearized models for the dynamics of the flow close to these steady states. These linearized models should be useful for control design, and so we include the effect of an actuator, which here is a streamwise body force in a small region near the leading edge of the airfoil.

The organization of this paper is as follows. In section [I] we discuss the numerical method used, a two-dimensional incompressible direct numerical simulation (DNS), using an immersed-boundary fractionalstep method developed by Taira and Colonius. ${ }^{7}$ Next, in section III, we compute steady states (both stable and unstable) and dominant eigenvectors of the flow past a flat plate at different angles of attack, using numerical wrappers around the DNS timestepper. Our system is too large to use conventional continuation packages such as AUTO ${ }^{8}$ which use direct linear solvers for the Jacobian matrix, so we use a timestepper based Newton-GMRES iteration to find these steady states.

Once the steady states are found, the equations are linearized about these states and reduced-order models are found using an approximation of balanced truncation that is tractable for systems with very large state dimension, ${ }^{9}$ described in section IV Balanced truncation is a model reduction method commonly used in the controls community for model reduction of stable linear systems, ${ }^{10}$ and has extensions to unstable ${ }^{11}$ and nonlinear ${ }^{12,13}$ systems as well. These reduced-order models are similar to models obtained by Proper Orthogonal Decomposition (POD),${ }^{14}$ but they have been shown to perform better than standard POD models, particularly when actuation is introduced..$^{9,15}$ We use these models to design an observer to reconstruct the flow state from a single surface pressure sensor, and perform a preliminary sensor-placement study using properties of the observability Gramian. A companion paper ${ }^{16}$ discusses three-dimensional simulations, and the effects of aspect ratio and planform shape on these dynamics.

\section{Numerical scheme}

The numerical scheme used is an immersed boundary fractional step method, ${ }^{7}$ which uses a finite volume scheme with spatial discretization on a staggered grid. The equations are integrated in time using an implicit Crank-Nicolson method for the viscous terms and an explicit, second order accurate Adams-Bashforth method for the convective terms. The body surface is treated as a set of Lagrangian points, with appropriate body forces that act as Lagrange multipliers to enforce the no-slip boundary condition. The form of the incompressible Navier-Stokes equations considered is

$$
\begin{aligned}
\partial_{t} u+u \cdot \nabla u & =-\nabla p+\frac{1}{\operatorname{Re}} \nabla^{2} u+\int f(\xi) \delta(\xi-x) d \xi \\
\nabla \cdot u & =0 \\
u(\xi) & =\int u(x, t) \delta(x-\xi) d x=u_{B},
\end{aligned}
$$


where $u$ and $p$ are velocity and pressure, $f$ is the force acting on the body surface $\xi$, which can move with a velocity $u_{B}$. Here, $u$ and $x$ are non-dimensionalized with respect to the free stream velocity $U$ and the flat plate chord length $c$, and the Reynolds number is defined as $\operatorname{Re}=U c / \nu$ where $\nu$ is the kinematic viscosity. The other quantities $p, f$, and time $t$ are consistently non-dimensionalized as well. We consider the body to be a stationary flat plate at an angle of attack $\alpha$. The discretization of the above equations yields

$$
\left[\begin{array}{ccc}
A & G & H \\
-D & 0 & 0 \\
E & 0 & 0
\end{array}\right]\left[\begin{array}{l}
q^{n+1} \\
p^{n+1} \\
f^{n+1}
\end{array}\right]=\left[\begin{array}{c}
r^{n}+b c_{1} \\
b c_{2} \\
u_{B}^{n+1}
\end{array}\right]
$$

where $G, D$ are the discrete gradient and divergence operators, and $A=\frac{1}{\Delta t} M-\frac{1}{2} L$, where $M$ is the mass matrix and $L$ is the discrete Laplacian. The explicit and boundary terms are contained in $r^{n}$ and $b c_{1,2}$ respectively, and $E, H$ are referred to as interpolation and regularization operators. ${ }^{7}$ In implementation, $p^{n+1}$ and $f^{n+1}$ are lumped together into a single variable $\lambda^{n+1}$ which acts as a Lagrange multiplier. Using an appropriate change of coordinates, the above equations can be solved using the following fractional step scheme:

$$
\begin{aligned}
A q^{*} & =r_{1}, & & \text { (Solve for the intermediate flux) } \\
Q^{T} B^{N} Q \lambda^{n+1} & =Q^{T} q^{*}-r_{2}, & & \text { (Solve the Poisson equation) } \\
q^{n+1} & =q^{*}-B^{N} Q \lambda^{n+1}, & & \text { (Projection step) }
\end{aligned}
$$

where $q$ is now the flux through the finite element boundaries, $Q=\left[G, E^{T}\right], B^{N} \approx A^{-1}$, and $r_{1}, r_{2}$ contain the effect of the explicit and boundary terms.

\section{Time-stepper based analysis of steady states}

Since our approach is to obtain reduced-order models of the flow linearized about a given steady state, we need to compute steady states and find dominant eigenvectors of the linearized transients. Of course, stable steady states can be computed by simply evolving the time-accurate simulation to stationarity. However, unstable steady states cannot be found in this manner, and stable steady states near a bifurcation point could take very long to converge. Instead, we use a Newton-GMRES ${ }^{17}$ method to compute steady states. We use a "timestepper-based" approach ${ }^{18,19}$ together with the original computational routine to compute the steady states using the dynamic simulator directly.

\section{A. Newton-GMRES method}

If the numerical time-stepper advances a velocity field $q^{k}$ at timestep $k$ to a velocity field $q^{k+T} \equiv \Phi_{T}\left(q^{k}\right)$ after $T$ timesteps, the steady state is given by the field $q^{*}$ that satisfies

$$
g\left(q^{*}\right)=q^{*}-\Phi_{T}\left(q^{*}\right)=0 .
$$

The steady states are given by zeros of $g\left(q^{*}\right)$, which could be solved for using Newton's method. However, the dimension of the system is too large to evaluate and invert full Jacobian matrices for this formulation. Instead of computing the Jacobian, we use GMRES, ${ }^{17,20}$ a Krylov space based iterative solver. This method requires computation of only Jacobian-vector products $D g(q) \cdot v$, which can be approximated using finite differences as $[D g(q+\epsilon v)-D g(q)] / \epsilon, \epsilon \ll 1$. A nice feature of GMRES is relatively fast convergence to the steady state when the eigenvalues of the Jacobian $D g\left(q^{*}\right)$ are clustered, ${ }^{17}$ which is often the case in the presence of multiple time-scales. ${ }^{19}$

The parameters for timestepper based steady-state computation were: grid size $=50 \times 50$, computational domain $=[-3,4] \times[-3.5,3.5]$, grid spacing in the vicinity of the flat plate $=0.08$, and time step $=0.04$. The Reynolds number used was 100, and steady states were computed for angles of attack varying from $\alpha=0$ to 30 in steps of $\alpha=0.5$. A plot of the steady state lift coefficient $C_{L}$ vs. $\alpha$ is given in Fig. 22a. Also plotted in the figure are the maximum and minimum values of $C_{L}$ for those angles of attack for which the stable flow consists of unsteady vortex shedding. We see that the (unstable) steady state values of $C_{L}$ are very close to the minimum value of the oscillatory case. Streamlines of representative steady states, both stable and unstable ( $\alpha=20$ and 25), are shown in Fig. 3 (left column, top and bottom). For comparison, the 
flow fields corresponding to the maximum and minimum oscillatory lift for $\alpha=25$ are also plotted (right column, top and bottom respectively). The fields with low values of $C_{L}$, that is, both the steady states and the minimum lift field in the unsteady case, have an attached trailing edge vortex, which is clearly absent in the maximum lift field.

For the Newton-GMRES computations, various values of the time reporting horizon $T$ in (8) were tried, ranging from 10 to 50. It was observed that increasing $T$ resulted in fewer GMRES iterations, and thus fewer calls to the time-stepper, the reason being an increased clustering of eigenvalues for larger $T .{ }^{19}$ However, since the number of integration steps per call to the timestepper also increases with $T$, the computational cost was found to remain more or less constant.

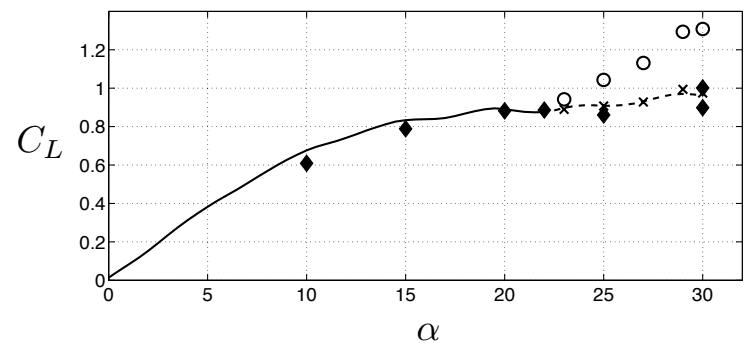

(a)

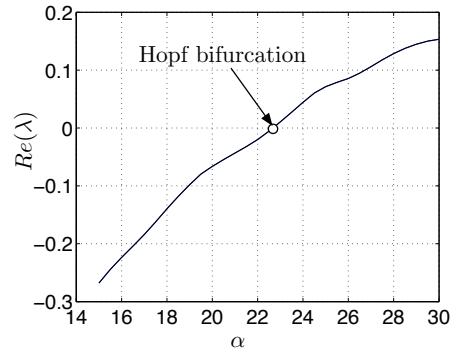

(b)

Figure 2. (a) $C_{L}$ vs. $\alpha$ at $\operatorname{Re}=100$. The solid line represents stable steady states and the dashed line represents unstable ones. Also shown are the maximum and minimum $C_{L}(\circ, \times)$ for the stable unsteady flow $(\alpha \gtrsim 23)$. The values obtained from a more highly resolved simulation, using parameters described in section C, are marked by $\$$. (b) Variation with $\alpha$ of the real part of the leading eigenvalue of the linearization about the steady states of plot (a).

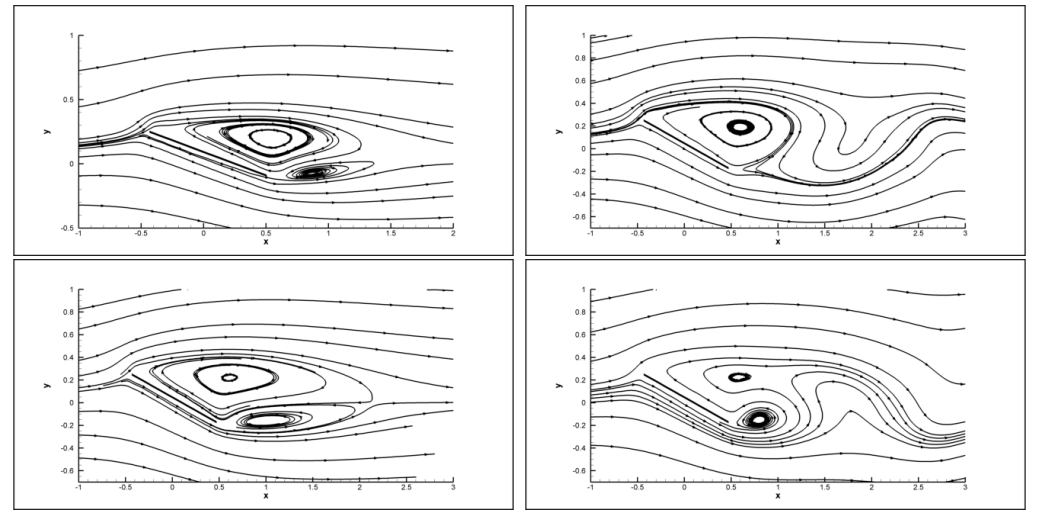

Figure 3. Streamlines of velocity fields. Steady states at $\alpha=20,25$ (left top and bottom). Unsteady fields at $\alpha=25$ corresponding to maximum and minimum (over an oscillation period) coefficients of lift (right top and bottom).

\section{B. Dominant eigenvectors}

The Newton-GMRES technique described above allows us to compute steady states, but gives no information regarding the stability of these steady states. For that, we need to compute the leading eigenvalues of the linearization about steady state, and track these along a solution branch to determine when a bifurcation occurs. We are also interested in obtaining reduced-order models for the flow linearized about unstable equilibria, as such models will be crucial to design stabilizing controllers. The approximate balanced truncation procedure outlined in the next section, originally developed for stable equilibria, can be extended to unstable systems as follows. Since the number of unstable directions is typically small, we can project out the flow in those directions. Then, we can obtain low order models for the stable subspace of the flow using POD/BPOD procedures. Now, in order to project out the unstable directions, we need to compute the right and the left eigenvectors of the linearization about a given steady state. We use a numerical library called ARPACK ${ }^{21}$ 
for this purpose. Like Newton-GMRES, ARPACK also uses a Krylov space technique and requires the computation of only Jacobian-vector products $D \Phi_{T}\left(u^{*}\right) \cdot v$, which we again compute using finite-differences. ARPACK can be used to locate a small number of eigenvalues with user-specified properties, such as the largest or smallest real part, largest or smallest magnitude, etc.

We used ARPACK in conjunction with our timestepper to compute the eigenvalues $\mu_{i}$ of linearization $D \Phi_{T}\left(u^{*}\right)$ about the branch of steady states shown in Fig. 2 a. These eigenvalues are related to those of the Jacobian of the (spatially discretized) differential operator by $\lambda_{i}=\log \mu_{i} / T$. The leading two eigenvalues form a complex pair, which crosses the imaginary axis from the left half complex plane at $\alpha \approx 22.5$ with non-zero speed, implying a super-critical Hopf bifurcation from a steady state to periodic vortex shedding; variation of the real part of this pair with $\alpha$ is plotted in Fig. $2 \mathrm{~b}$. We also computed the corresponding eigenvectors, and the real and imaginary parts for $\alpha=25$ are plotted in Fig. 4 . These appear qualitatively similar to the POD modes of the transients close to the corresponding steady state, as one might expect.

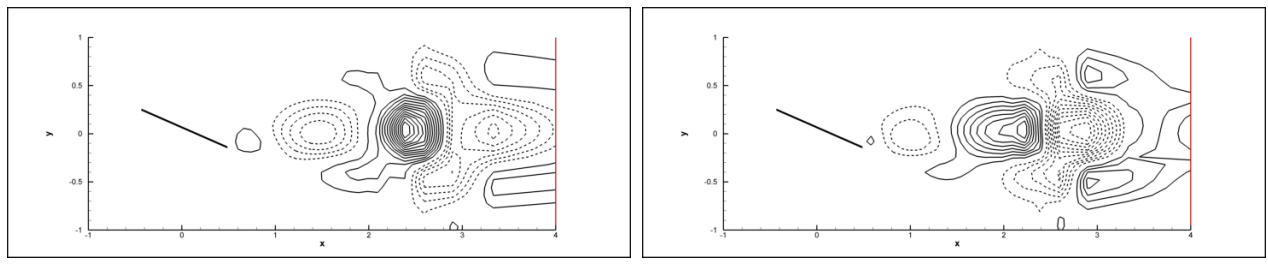

Figure 4. Real and imaginary part of the leading eigenvector of the linearization about the unstable steady state at $\alpha=25$. Vorticity contours are plotted.

\section{Reduced-order models}

\section{A. Balanced Proper Orthogonal Decomposition}

The procedure we use to obtain reduced-order models is a numerical approximation to balanced truncation, a method commonly used to reduce the dimension of controllers. ${ }^{10,22}$ Balanced truncation gives reducedorder models for stable, linear input-output systems, with operator-norm error bounds that are close to the minimum achievable for any reduced-order model. For systems with more than $\approx 1000$ states, however, conventional procedures for computing balanced realizations are not computationally tractable (they scale as $n^{3}$ ), so here we use a numerical approximation we call Balanced Proper Orthogonal Decomposition, ${ }^{9}$ which has computational cost comparable to that of the conventional POD/Galerkin procedure.

One begins with a stable input-output system of the form

$$
\begin{aligned}
x^{n+1} & =A x^{n}+B u^{n} \\
y^{n} & =C x^{n}
\end{aligned}
$$

where $x^{n}$ is the state vector, $u^{n}$ is a vector of inputs, $y^{n}$ is a vector of outputs, and $n$ indicates the timestep. Here, $x$ consists of the velocities at each gridpoint, and $C$ is the identity matrix, so the output is the entire state. The balanced POD procedure proceeds as follows:

1. Compute the (state) response of the linearized equations to an impulse on each input.

2. Assemble the resulting snapshots, and compute POD modes $\theta_{j}$ of the resulting dataset.

3. Choose the number of POD modes one wants to use to describe the output of the system. For instance, if $10 \%$ error is acceptable, and the first $r$ POD modes capture $90 \%$ of the energy, then keep the first $r$ modes.

4. Compute the (state) response of the adjoint equations $z^{n+1}=A^{T} z^{n}$, starting with each POD mode $\theta_{j}$ as the initial condition (one simulation for each of the first $r$ modes).

5. If $x^{n}$ are the snapshots from step 1, and $z^{m}$ are the snapshots from step 4. construct the matrices $X$ with $x^{m}$ as columns, $Y$ with $z^{n}$ as columns, and $M=Y^{T} X$ (that is, $M_{m n}=\left\langle x^{n}, z^{m}\right\rangle$ ), and compute the singular value decomposition $M=U \Sigma V^{T}$. 
6. Define balancing modes $\varphi_{j}$ and the corresponding adjoint modes $\psi_{j}$ as columns of the matrices $\Phi, \Psi$, where

$$
\Phi=X V \Sigma^{-1 / 2}, \quad \Psi=Y U \Sigma^{-1 / 2} .
$$

Then the modes $\varphi_{j}$ and $\psi_{j}$ are bi-orthogonal (i.e., $\left\langle\varphi_{j}, \psi_{k}\right\rangle=\delta_{j k}$ ), and a reduced-order model of $(9)$ is given by

$$
\begin{aligned}
a^{n+1} & =\Psi^{T} A \Phi a^{n}+\Psi^{T} B u^{n} \\
y^{n} & =C \Phi a^{n} .
\end{aligned}
$$

For more details on this method and its relation to the standard POD/Galerkin procedure, see Rowley. ${ }^{9}$

\section{B. Linearized and adjoint models}

The linearized equations are obtained by replacing the convective term in (1) with $U \cdot \nabla u+u \cdot \nabla U$, where $U$ is the steady state. These equations can be easily solved using the immersed boundary scheme (5 77 by appropriately modifying the convective terms in $r_{1}$ and $r_{2}$. The linearization of the terms $r_{1}$ and $r_{2}$ are denoted by $L_{1} q^{n}$ and $L_{2} q^{n}$ respectively, where $L_{1}$ and $L_{2}$ are appropriate linear operators that depend on the steady state $U$.

For obtaining low order models using balanced truncation, we also need to solve adjoint equations. We construct the adjoint of the discrete equations (5 7) for reasons of simplicity and numerical consistency of the procedure. These equations can also be expressed in a fractional step scheme as follows:

$$
\begin{aligned}
Q^{T} B^{N} Q \lambda^{n+1} & =Q^{T} B^{N} q^{n} \\
A q^{*} & =q^{n}-Q \lambda^{n+1}, \\
q^{n+1} & =L_{1}^{*} q^{*}+L_{2}^{*} \lambda^{n+1},
\end{aligned}
$$

where $L_{1}^{*}$ and $L_{2}^{*}$ are the discrete adjoints of $L_{1}$ and $L_{2}$.

\section{Results}

We restrict our attention to the case of $\alpha=20$ and $\operatorname{Re}=100$, which is in the regime of stable steady flow. The computational parameters used for numerical simulations of the linearized and adjoint equations were: grid size $=250 \times 200$, computational domain $=[-5,10] \times[-1010]$, grid spacing in the vicinity of the flat plate $=0.02$, and time step $=0.01$. These parameters result in a much finer simulation as compared to that in section III. For this grid size, the number of states in the simulation was approximately $2 \times 10^{5}$ which is intractable for control design using traditional techniques. Hence, we obtain reduced-order models using the snapshot-based balanced truncation technique described in section IV and compare them to the standard POD-based models.

The actuator considered for computing the impulse response of the linearized equations was a localized body force acting in the region of the flow behind the leading edge of the plate; the vorticity contours of the flow field immediately after an impulsive input are plotted in Fig. 5 a. This actuator could be thought of as a simple model of a pulse blowing upstream or a disturbance in the flow.

In the impulse response, the disturbance convects downstream and triggers transient vortex shedding in the wake, which eventually decays away. We used 700 snapshots spaced 5 timesteps apart for computing the POD modes of this flow. As seen from Fig. $5 \mathrm{~b}$, the energy content of the modes quickly drops; the first four modes contain almost $84 \%$, while the first eight modes contain almost $92 \%$ of the total energy. Also, these modes occur in pairs in terms of their energy content, as is typically observed in periodic flows. However, the structure of modes is different from that reported in other periodic flows, where the higher modes are harmonics of the first pair. The vorticity contours of modes 1, 3, and 5 are plotted in Fig. 6. From these plots, we observe that the spatial wavelength of the wake structures is the same in these modes, while these structures are located further downstream in the modes with greater energy content.

Since most of the energy is contained in the first four modes, we consider the output $y$ to the a projection of the state $x$ onto the first four POD modes and compute the approximate balancing transformation. The adjoint snapshots are obtained from four adjoint simulations with each of the first four POD modes as an initial condition. The Hankel singular values of the resulting balancing transformation, shown in Fig. 5r, also occur in pairs, and drop off quickly allowing a low order truncation. The balancing and the adjoint 


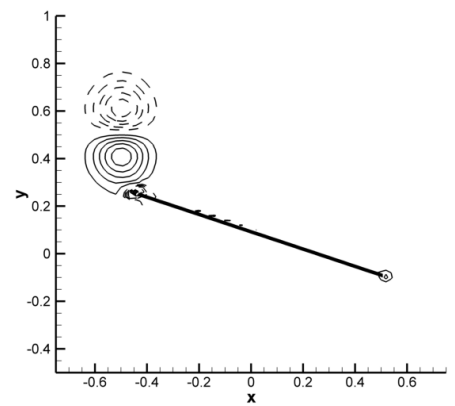

(a)

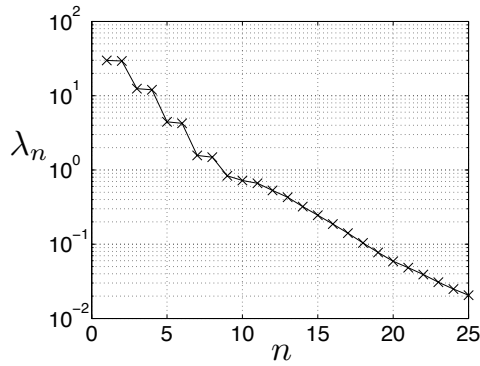

(b)

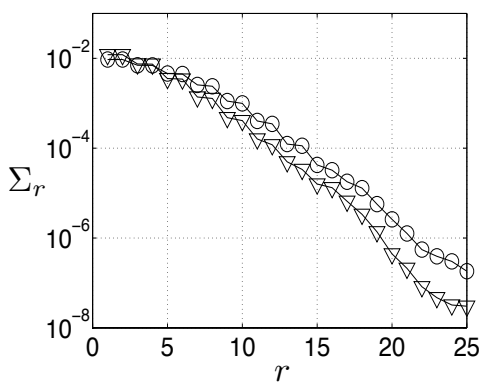

(c)

Figure 5. (a) Vorticity contours of the flow field immediately after an impulsive input. Negative contours are plotted in dashed lines. (b) Energy content of the POD modes of the impulse response. (c) Hankel singular values of the approximate balancing transformation with the output being a projection onto the leading two $(\nabla)$ and four (o) POD modes.
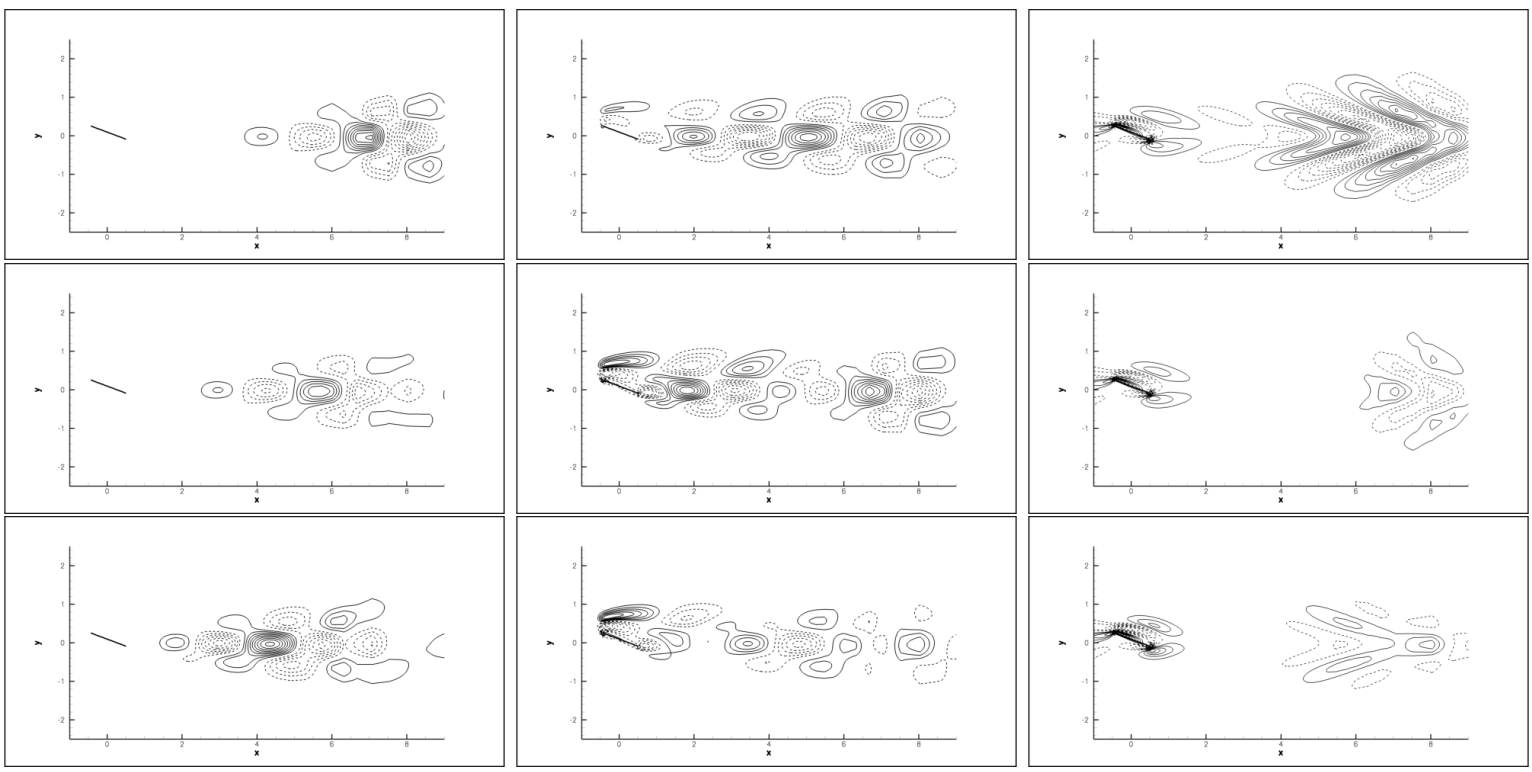

Figure 6. Vorticity contours of the POD (left), balancing (center), and adjoint modes (right) of the impulse response. Negative contours are in dashed lines. The modes ranked 1, 3, and 5 (from top to bottom) by energy for POD and by Hankel singular values for balanced truncation are shown. 
modes are shown alongside the POD modes in Fig. 6, where vorticity contours are plotted for the balancing modes, and of the corresponding "vorticity-like" variable for the adjoint modes. Both these sets of modes look different from the POD modes, in the sense that the POD modes have almost no support in the nearwake region, while the balancing and the adjoint modes both highlight this region considerably. We quantify this difference by comparing the subspaces spanned by the POD and the balancing modes as follows. We compute the quantity trace $\left(P_{r} \widetilde{P}_{n}\right)$, where $P_{r}$ is an orthogonal projection onto the first $r$ POD modes, and $\widetilde{P}_{n}$ is an orthogonal projection onto the first $n$ balancing modes. This quantity gives a measure of how well the first $n$ balancing modes span the subspace formed by the first $r$ POD modes. ${ }^{23}$ Its upper bound is $r$ and is achieved only when the POD subspace is completely spanned by the balancing modes. As seen from Fig. 7 a, at least 4, 8, 12, and 16 balancing modes are required to span the subspace formed by the first 2,4 , 6 , and 8 POD modes respectively.

Next, we examine how well the two sets of modes capture the subspace spanned by the actuators (i.e., the columns of the matrix $B$ ). To quantify this, we compute the quantity trace $\left(P_{B} P_{r}\right)$, where $P_{B}$ is a projection onto the actuator subspace, which in our case is just one dimensional. If orthogonal projections are used, this quantity may be interpreted geometrically as the cosine of the angle between the actuation direction and the subspace spanned by the first $r$ modes. Fig. $7 \mathrm{~b}$ shows that the first eight POD modes do not capture the actuator at all (actually the projection is not zero, but close to zero), and thus the resulting low order models cannot capture the impulse response to this actuation. On the other hand, the subspace spanned by the balancing modes is comparatively much closer to the actuation subspace. This will lead to better dynamic performance of the low order models, as has been shown for the case of a 3-D channel flow. ${ }^{15}$

The figure also shows the result for a non-orthogonal projection, using the adjoint modes: that is, $P_{r}=\Phi_{r} \Psi_{r}^{T}$, where $\Phi_{r}$ and $\Psi_{r}$ are the first $r$ columns of $\Phi, \Psi$ in 10 . It is clear that using the adjoint modes for projection, as done in the formulation of the reduced-order models, significantly affects the projection, and captures the actuation much better.

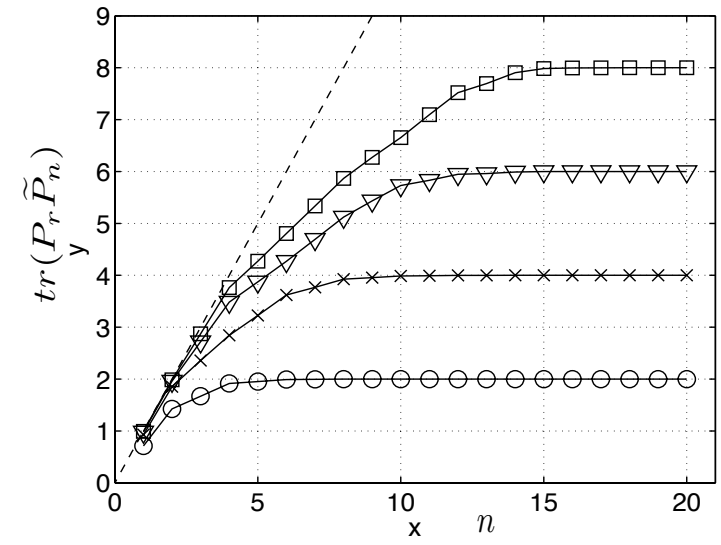

(a)

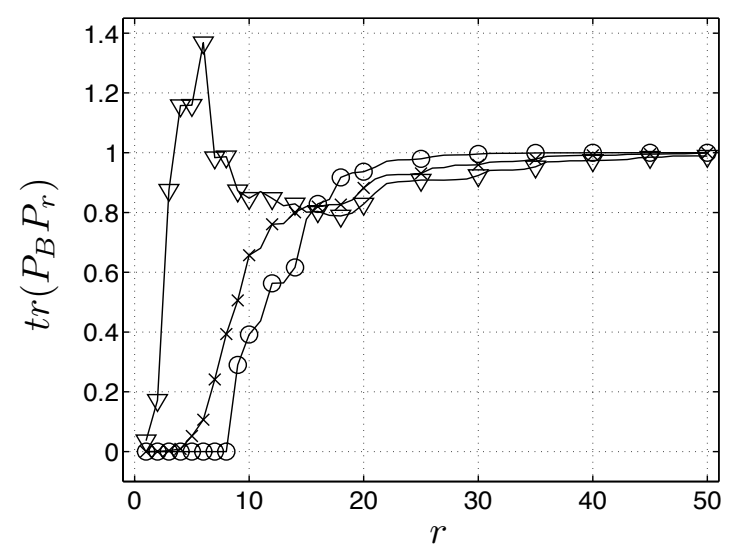

(b)

Figure 7. (a) Measure of distance between the subspaces formed by the first $n$ balancing modes and the first $r$ POD modes. Plots of $\operatorname{tr}\left(P_{r} \widetilde{P}_{n}\right)$ vs. $n$ for $r=2,4,6,8(\circ, \times, \nabla, \square)$. (b) An orthogonal projection of the flow field after an impulsive input onto the first $r$ balancing $(\times)$ and POD ( $\circ)$ modes. Also shown is a non-orthogonal projection onto the first $r$ balancing modes $(\nabla)$, using the adjoint modes for the projection.

Some representative results of the low order models are presented in Fig. 8. The figure compares the projection of the DNS data onto the first and third POD modes with the predictions of POD and balancedPOD models. The plots show that a 12-mode balanced-POD model predicts the output accurately. A 12 -mode POD model under-predicts the outputs, while a 16 -mode POD model is accurate until $t \approx 25$. For later times, the 16-mode POD model incorrectly predicts a large growth of the outputs (the model is stable, but barely), whereas the actual response gradually decays to zero. Note that the amplitudes of the outputs close to initial time are very small, indicating that the projection of the actuator subspace onto the first few POD modes is small, consistent with our previous findings. 

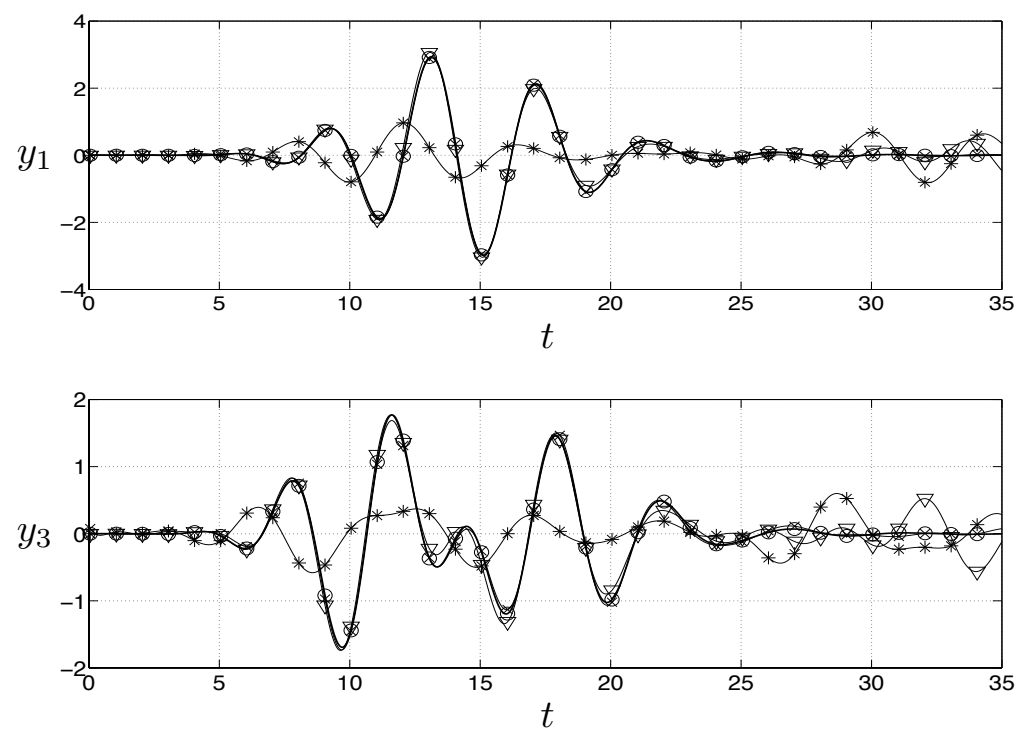

Figure 8. Comparison of outputs $y_{1}$ and $y_{3}$ of the reduced order models with projection of snapshots onto the POD modes (०). 12-mode POD $(*)$, 16-mode POD $(\nabla)$, and 12-mode balanced POD $(\times)$.

\section{Full-state reconstruction}

Since it is usually not possible to measure the entire velocity field in practical applications, it is desirable to reconstruct it from a few sensor measurements. Here, we use the reduced order models derived in section IV to design observers using one pressure measurement on the surface of the flat plate. The output equation in 11 is replaced by the following:

$$
\tilde{y}^{n}=p^{n}=\sum_{i=1}^{N} \zeta_{i} a_{i}^{n} \stackrel{\text { def }}{=} \widetilde{C} a^{n},
$$

where $\zeta_{i}$ are the pressures (at the sensor location) corresponding to the balancing modes $\phi_{i}$. (This approach to obtaining the output equation was also used by Rowley and Juttijudata. ${ }^{24}$ ) We consider two different sensor locations for observer design, one above the leading edge, and the other above the trailing edge of the plate. As a first step, in order to decide the number of modes required in the reduced order model for a good observer design, we compare the output as given by 15 with the pressure measurements from the full simulation of the linearized equations. As shown in Fig. 9, while 12 modes are required for a faithful reconstruction of the trailing edge pressure trace, as many as 20 modes are needed for the accurate leading edge pressure trace reconstruction.

We design an observer for the model (11) of the form

$$
\hat{a}^{n+1}=\Psi^{T} A \Phi \hat{a}^{n}+\Psi^{T} B u^{n}+L\left(y^{n}-\widetilde{C} \hat{a}^{n}\right),
$$

where, $\hat{a}^{n}$ is an estimate of the state $a^{n}$, and $L$ is the observer gain obtained using standard linear control techniques such as pole placement or LQE.

We will now discuss the performance of different observers using the plots of reconstruction of the first state $a_{1}$ shown in Fig. 10 Since there is no output information available at the beginning of a simulation, the initial estimate $\hat{a}^{0}$ is always taken to be zero. As shown in Fig. 10a , the observer based on a trailing edge sensor reconstruct the state very well; the one based on a 12-mode model performs well till $t \approx 20$, after which it overpredicts the amplitude of the oscillations. This is due to the poor reconstruction of the pressure using 12 modes, as was seen in Fig. 9 . The observer based on a 20-mode model, on the other hand, tracks the state accurately for the entire length of time considered. With a leading edge sensor, the performance is poor even with a 20-mode model, as can be seen from Fig. 10p. The amplitude of the reconstructed state is initially under-predicted for $t \lesssim 20$, and is subsequently over-predicted for later times. That can again 


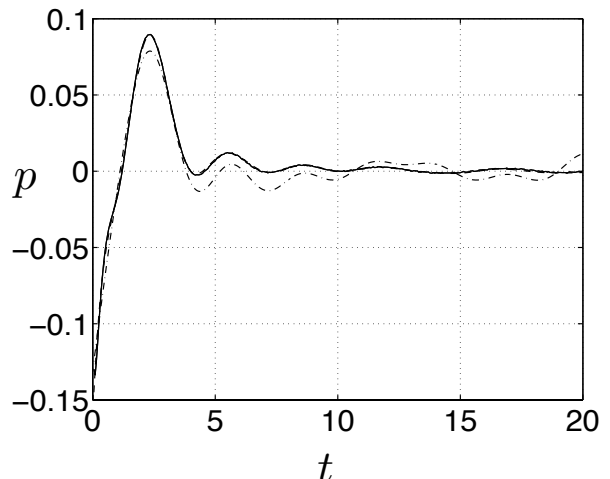

(a)

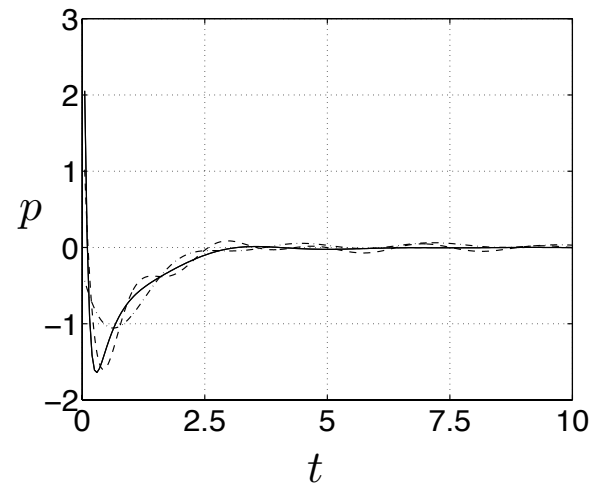

(b)

Figure 9. Pressure traces at (a) trailing and (b) leading edges (in solid line), and their reconstruction using a 12-mode $(-\cdot-)$ and a 20-mode $(--)$ model.

(a)
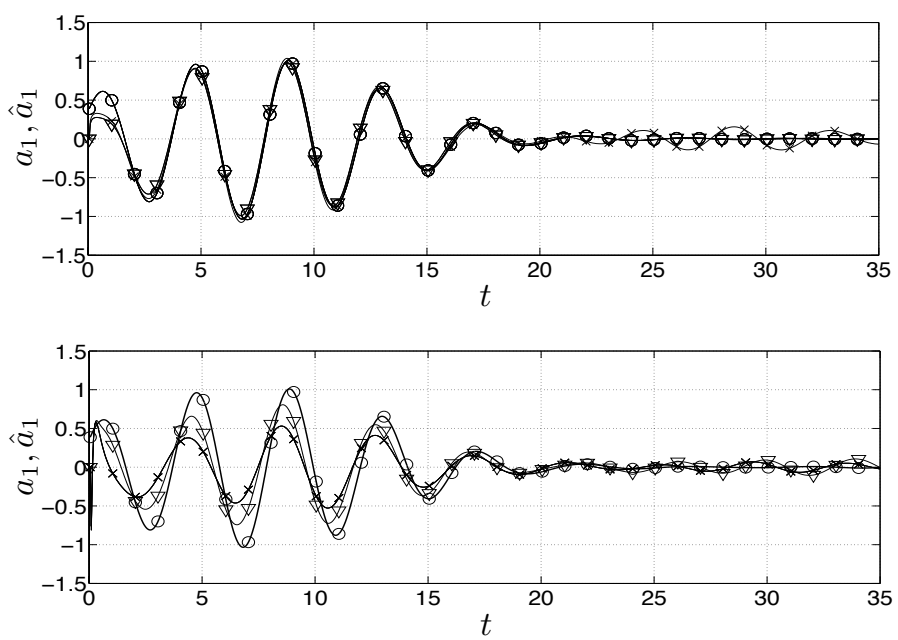

Figure 10. Reconstruction of the first state using observers based on reduced order models: the actual state $a_{1}$ $(\circ)$ is compared to estimates $\hat{a}_{1}$ from a 12-mode observer $(\times)$ and a 20-mode observer ( $\left.\nabla\right)$. (a) Sensor at the trailing edge; (b) Sensor at the leading edge. 
be explained using the fact that the reconstruction of pressure using this sensor is not accurate, as noted earlier.

We now give an alternative explanation for the relatively poor performance of the leading edge sensor. Note that when the output equation (9) is replaced by (15), the resulting realization is usually not balanced. That is, the most controllable directions (which for this realization are the first few states) need not be the most observable directions. We compute the observability Gramian $W_{o}$ corresponding to the pair $\left(\Psi^{T} A \Phi, \widetilde{C}\right)$. Then we compare the subspaces spanned by the first few (say 6) controllable and observable directions by using the metric used earlier in section C. The values of this metric for sensors located at various points on the plate (given in Table 1) are much smaller than 6, which is the upper bound achieved in case of identical subspaces. This implies that the resulting systems are far from balanced in all the cases. However, the subspaces are much closer for the trailing edge sensor as compared to the other locations. These ideas could be used for optimizing the sensor locations.

\begin{tabular}{cccccc}
\hline Sensor location $(l / c)$ & 0 & 0.25 & 0.5 & 0.75 & 1.0 \\
\hline $\operatorname{trace}\left(P_{C} P_{O}\right)$ & 0.0080 & 0.0053 & 0.0654 & 0.0226 & 0.1853 \\
\hline
\end{tabular}

Table 1. Comparison of subspaces formed by the six most controllable and observable directions, for different sensor locations ( $l$ denotes distance from the leading edge); $P_{C}$ and $P_{O}$ are orthogonal projections onto these subspaces respectively. Values must lie between 0 (orthogonal subspaces) and 6 (identical subspaces).

\section{Conclusions}

We have described reduced-order models for the influence of a body-force actuator on the flow past a flat plate. A timestepper-based Newton-GMRES iteration was used to find both the stable and the unstable steady states as the angle of attack was varied, and the dominant eigenvectors were found using ARPACK. A super-critical Hopf bifurcation is observed as the angle of attack increases, and for the stable case, the dominant eigenvectors are close to the dominant POD modes of the impulse response, as expected.

We then developed reduced-order models of the flow linearized about a stable steady state, using the balanced POD (BPOD) procedure. This method requires simulations of both the linearized and adjoint equations, and the overall computational effort required is comparable to the standard POD/Galerkin method. The BPOD models perform much better than the standard POD models: they capture transients more accurately, and all of the models computed were stable, unlike standard POD models, which sometimes have the wrong stability type. The subspaces spanned by the balancing modes are close to those spanned by standard POD modes, but the adjoint modes used in the projecting the dynamics look quite different. These adjoint modes have large magnitude close to the flat plate, where the actuator is located, and as a result the BPOD models capture the effects of the actuator much more effectively than the standard POD models. The resulting models were used to design a dynamic observer that effectively reconstructs the flow state from a single pressure measurement at the wall, and trailing-edge sensors were found to perform better than leading-edge sensors for the case studied.

Future studies will address models near unstable steady states, explore the sensor-placement problem in more detail, and use the types of models presented here for closed-loop control design.

\section{Acknowledgments}

We gratefully acknowledge Sam Taira for help with the immersed boundary simulations, and Liang Qiao for help with timestepper based analysis of steady states. This research was supported by AFOSR, grants FA9550-05-1-0369 and FA9550-06-1-0371.

\section{References}

\footnotetext{
${ }^{1}$ Johnson, W., Helicopter Theory, Princeton University Press, 1980.

${ }^{2}$ Magill, J., Bachmann, M., Rixon, G., and McManus, K., "Dynamic stall control using a model-based observer," J. Aircraft, Vol. 40, No. 2, 2003, pp. 355-362.

${ }^{3}$ Smith, A. C. and Baillieul, J., "Vortex models for the control of stall," Proceedings of the 42nd IEEE CDC, WeM12-5,
} 
2003, pp. 2407-2412.

${ }^{4}$ Smith, A. C., Vortex models for the control of stall, Ph.D. thesis, Boston University, 2005.

${ }^{5}$ Liiva, J. and Davenport, F. J., "Dynamic stall of airfoil sections for high-speed rotors," J. Amer. Helic. Soc., Vol. 14, No. 2, 1969, pp. 26-33.

${ }^{6}$ Birch, J. M. and Dickinson, M. H., "Spanwise flow and the attachment of the leading-edge vortex on insect wings," Nature, Vol. 412, Aug. 2001, pp. 729-733.

${ }^{7}$ Taira, K. and Colonius, T., "The Immersed Boundary Method: A Projection Approach," J. Comput. Phys., submitted,

${ }^{8}$ Doedel, E. J., Keller, H. B., and Kernevez, J. P., "Numerical analysis and control of bifurcation problems, Part I," Int. J. Bifurcation Chaos, Vol. 1, No. 3, 1991, pp. 493-520.

${ }^{9}$ Rowley, C. W., "Model reduction for fluids using balanced proper orthogonal decomposition," Int. J. Bifurcation Chaos, Vol. 15, No. 3, March 2005, pp. 997-1013.

${ }^{10}$ Moore, B. C., "Principal Component Analysis in Linear Systems: Controllability, Observability, and Model Reduction," IEEE Trans. Automat. Contr., Vol. 26, No. 1, Feb. 1981, pp. 17-32.

${ }^{11}$ Zhou, K., Salomon, G., and Wu, E., "Balanced realization and model reduction for unstable systems," Int. J. Robust and Nonlin. Contr., Vol. 9, No. 3, March 1999, pp. 183-198.

${ }^{12}$ Lall, S., Marsden, J. E., and Glavaški, S., "A subspace approach to balanced truncation for model reduction of nonlinear control systems," Int. J. Robust Nonlinear Control, Vol. 12, 2002, pp. 519-535.

${ }^{13}$ Scherpen, J. M. A., "Balancing for nonlinear systems," Sys. Control Lett., Vol. 21, No. 2, 1993, pp. $143-153$.

${ }^{14}$ Holmes, P., Lumley, J. L., and Berkooz, G., Turbulence, Coherent Structures, Dynamical Systems and Symmetry, Cambridge University Press, Cambridge, UK, 1996.

${ }^{15}$ Ilak, M. and Rowley, C. W., "Reduced-order modeling of channel flow using traveling POD and balanced POD," AIAA Paper 2006-3194, 3rd AIAA Flow Control Conference, June 2006.

${ }^{16}$ Taira, K., Dickson, W. B., Colonius, T., Dickinson, M. H., and Rowley, C. W., "Unsteadiness in flow over a flat plate at angle-of-attack at low Reynolds numbers," AIAA Paper 2007-710, 45th AIAA Aerospace Sciences Meeting and Exhibit, Jan. 2007.

${ }^{17}$ Kelley, C. T., Iterative methods for linear and nonlinear equations, No. 16 in Frontiers in Applied Mathematics, SIAM, 1995.

${ }^{18}$ Tuckerman, L. and Barkley, D., "Bifurcation analysis for timesteppers," Vol. 19, No. 119, 1999, pp. 453-466.

${ }^{19}$ Kelley, C. T., Kevrekidis, I. G., and Qiao, L., "Newton-Krylov solvers for timesteppers," Preprint at arXiv.org (math.DS/0404374), 2004.

${ }^{20}$ Saad, Y. and Schultz, M. H., "GMRES: A generalized minimal residual algorithm for solving nonsymmetric linear systems," SIAM J. Sci. Statist. Comput., Vol. 7, No. 3, 1986, pp. 856-869.

${ }^{21}$ Lehoucq, R. B., Sorensen, D. C., and Yang, C., ARPACK Users' Guide, SIAM, 1998.

${ }^{22}$ Cortelezzi, L., Lee, K. H., Kim, J., and Speyer, J. L., "Skin-friction Drag Reduction via Robust Reduced-order Linear Feedback Control," Int. J. Comput. Fluid Dyn., Vol. 11, 1998, pp. 79-92.

${ }^{23}$ Everson, R. and Sirovich, L., "Karhunen-Loève procedure for gappy data," J. Opt. Soc. Am. A, Vol. 12, No. 8, Aug. 1995, pp. $1657-1664$.

${ }^{24}$ Rowley, C. W. and Juttijudata, V., "Model-based control and estimation of cavity flow oscillations," Proceedings of the 44th IEEE Conference on Decision and Control, Seville, Spain, Dec. 2005. 